- Original Article

\title{
Association between Sleep Duration and Body Composition Measures in Korean Adults: The Korea National Health and Nutrition Examination Survey 2010
}

\author{
Ryoung Hee Kim, Kyong In Kim, Jeong Hyeon Kim*, Yong Soon Park \\ Department of Family Medicine, Hallym University Chuncheon Sacred Heart Hospital, Chuncheon, Korea
}

\begin{abstract}
Background: The association between sleep duration and body composition measures in Korean adults remains unclear.

Methods: This cross-sectional study included 3,532 subjects aged $\geq 40$ years (1,542 men and 1,990 women) who participated in the 2010 Korea National Health and Nutrition Examination Survey V-1. Self-reported sleep duration and anthropometric data were collected. Appendicular skeletal muscle mass (ASM) was quantified via dual-energy X-ray absorptiometry. Obesity was defined according to the body mass index and waist circumference. Sarcopenia was defined as the muscle mass percentage (ASM/weight) below the lowest quintile computed for the study population. Multivariate logistic regressions with or without adjustment for sociodemographic and lifestyle factors were used to evaluate the association of sleep duration with obesity and sarcopenia for participants who slept $\leq 5,6-8$, and $\geq 9 \mathrm{~h} / \mathrm{d}$. The results were expressed as odds ratios (ORs) with $95 \%$ confidence intervals (95\% CIs).

Results: In women, the association between sleep duration and sarcopenia was stronger for individuals who slept $\geq 9 \mathrm{~h} / \mathrm{d}$ than for those who slept 6-8 $\mathrm{h} / \mathrm{d}$ (unadjusted OR, 1.99; 95\% CI, 1.19-3.34; adjusted OR, 1.77; 95\% CI, 1.062.96).

Conclusion: Longer sleep duration is associated with a significantly higher incidence of sarcopenia in Korean women aged $\geq 40$ years.
\end{abstract}

Keywords: Sleep; Sarcopenia; Obesity; Korea National Health and Nutrition Examination Survey

Received: March 2, 2017, Revised: April 24, 2017, Accepted: May 7, 2017

${ }^{*}$ Corresponding Author: Jeong Hyeon Kim https://orcid.org/0000-0002-8889-4437

Tel: +82-33-240-5311, Fax: +82-33-240-5440, E-mail: next1203@hanmail.net 


\section{INTRODUCTION}

Normal aging is associated with changes in body composition characterized by a relative decline in muscle mass and a progressive increase in fat mass. ${ }^{1)}$ Body composition redistribution begins to accelerate in the late $30 \mathrm{~s}$ and into the $40 \mathrm{~s}^{2)}$ Obesity, which consists of abnormal or excessive fat accumulation, is known to be associated with an increased risk of mortality and adverse health outcomes, including cancer, cardiovascular disease, diabetes, and hypertension. ${ }^{3)}$ Sarcopenia, which is the age-related progressive loss of muscle mass, is also known to be associated with adverse health outcomes, such as physical inactivity, disability, diabetes, metabolic syndrome, cardiovascular disease, and mortality. ${ }^{4,5)}$ Considering the significant influence of body composition on the health of middle-aged and elderly individuals, it is of great relevance to determine the factors related to changes in body composition and to understand their mechanisms of action in the general population.

Sleep is considered an important lifestyle factor affecting health. Not only are sleep disorders such as sleep loss, long-term sleep deprivation, and alterations in sleep duration common in modern society, but the risks associated with sleep disorders increase with age. ${ }^{6,7)}$ In particular, physical inactivity, lower functional capacity, and physical disability are associated with longer sleep duration. ${ }^{8,9)}$ Additionally, several studies have reported associations between sleep duration (shorter or longer) and obesity. However, given the variations in study design and large differences in the target populations, it is difficult to draw conclusions on the consistency of these associations. Moreover, most studies on this topic have been conducted in Western populations, which differ from Asian populations in terms of sleep patterns, body composition, and relevant sociocultural factors ${ }^{10,11)}$ such as occupational stress and eating habits, which may significantly affect the relationship between sleep and body composition measures.

Despite the enormous clinical importance of sleep and body composition, very few studies have assessed the effects of sleep duration on obesity and the sarcopenic status in the general population. This paucity of data is also apparent in Korean populations. Furthermore, the redistribution of body composition, including aspects involved in obesity and the sarcopenic status, is known to accelerate and progress substantially in the late 30 s and into the $40 \mathrm{~s}^{2}{ }^{2}$ However, previous studies conducted in Korea have not focused on subjects aged 40 years or older, i.e., those expected to experience accelerated redistribution of body composition. In addition, previous studies undertaken in Korea considered only obesity indices, and not sarcopenia. ${ }^{12)}$ Therefore, the present study, which was conducted using nationally representative survey data, aimed to assess the relationship of sleep duration with obesity and sarcopenia in Korean adults aged 40 years or older.

\section{METHODS}

\section{Study Population}

The study was based on the 5th Korea National Health and Nutrition
Examination Survey (KNHANES V-1), conducted in 2010 by the Korea Centers for Disease Control and Prevention, which aimed to use the data for estimating health status in the Korean population and evaluating health policies and programs. All study participants provided informed consent, and the appropriate ethics review board of the Chuncheon Sacred Heart Hospital approved the study design (IRB approval no., 2017-32). KNHANES enrolled representative subjects selected by stratified multistage sampling. These surveys have generated nationwide and representative statistical data through self-administered questionnaires regarding health status, nutritional status, and health behaviors. $^{13)}$

The Korea Centers for Disease Control and Prevention selected 10,983 individuals from households of various sizes $(n=3,840)$. Of these, 8,958 subjects participated in the KNHANES V-1 surveys (response rate $=81.9 \%$ ). We applied the following exclusion criteria: age $<40$ years $(n=4,406)$; previous diagnosis of ischemic heart disease, stroke, liver cirrhosis, chronic kidney disease, or cancer $(n=479)$; and missing data $(n=541)$. A total of 3,532 subjects $(1,542$ men and 1,990 women) were included in the final statistical analysis.

\section{Definitions of Obesity and Sarcopenia}

Height and body weight were obtained according to the standard guidelines using internationally certified anthropometric equipment. Height $(\mathrm{cm})$ was measured with participants standing upright and not wearing shoes. Body weight $(\mathrm{kg})$ was measured with participants dressed in light clothing but not wearing shoes. Waist circumference (cm) was measured at the midpoint between the lower end of the 12th rib and the upper end of the iliac crest during minimal respiration. The body mass index (BMI) was calculated as body weight $(\mathrm{kg})$ divided by height ( $\mathrm{m}$ ) squared (i.e., $\mathrm{kg} / \mathrm{m}^{2}$ ). A cutoff of BMI $\geq 25 \mathrm{~kg} / \mathrm{m}^{2}$ was used to define general obesity, while central obesity was defined as a waist circumference $\geq 90 \mathrm{~cm}$ in men and $\geq 85 \mathrm{~cm}$ in women. ${ }^{14)}$

Body composition, including body fat percentage and appendicular skeletal muscle mass (ASM, in kg), were quantified using dual-energy X-ray absorptiometry (DISCOVERY-W fan-beam densitometer; Hologic Inc., Bedford, MA, USA). ASM was defined as the sum of the muscle mass in the arms and legs, assuming that all non-fat and nonbone tissues were skeletal muscles. The sarcopenic status was defined as the percentage of muscle mass (ASM/weight) below the lowest quintile computed for the study population, based on the data and recommendations included in the consensus report of the Asian Working Group for Sarcopenia. ${ }^{15)}$ Therefore, in the present study, the cutoff for sarcopenic status was a muscle mass percentage of $29.26 \%$ in men and $29.54 \%$ in women.

\section{Sleep Duration and Other Variables}

Sleep duration (h/d) was self-reported in integer values. Specifically, the subjects reported a number between 00 and 24 hours in response to the question "How long do you usually sleep at night?" The responses were classified into three categories: short sleep duration $(\leq 5$ $\mathrm{h} / \mathrm{d}$ ), appropriate sleep duration (6-8 h/d), and long sleep duration ( $\geq 9$ 
h/d)..$^{12,16,17)}$

Household equivalent income was calculated as the sum of the monthly income of all household members divided by the square root of the number of household members. Subsequently, household equivalent income was classified into four groups: lowest, middle-low, middle-high, and highest. Regular exercise was defined as having occurred in respondents who performed moderate-to-strenuous exercise on a regular basis (i.e., moderate exercise for $>30$ minutes at a time, $>5$ times per week; or strenuous exercise for $>20$ minutes at a time, $>5$ times per week). Smoking status was defined as non-smoker or current smoker based on the answer to the question "Do you currently smoke?" Alcohol drinking status was assigned based on the answer to the question "Did you drink more than one glass of liquor during the last month?"13)

\section{Statistical Analysis}

To minimize the bias in the representation of the general Korean population, all estimates were calculated based on sample weights and adjusted for the complex sample design of the survey. Data for continuous and categorical variables were expressed as mean \pm standard error (SE) and percentage $( \pm \mathrm{SE})$, respectively. General characteristics were summarized according to sleep duration and sex, and betweengroup differences were assessed using analysis of variance and chisquare tests. Odds ratios (ORs) and 95\% confidence intervals (CIs) for the associations of sleep duration with obesity and sarcopenia by sex were estimated using multivariate logistic regression analyses with or without adjustment for confounding factors. Statistical analyses were performed using SAS ver. 9.3 (SAS Institute Inc., Cary, NC, USA) with the survey sampling procedure. All tests were two-sided, and P-values $<0.05$ were considered to indicate statistical significance.

\section{RESULTS}

The mean age in the male and female respondents was $53.7 \pm 0.4$ and
$55.7 \pm 0.4$ years, respectively. Approximately $56.3 \%(n=1,990)$ of the respondents were women (Table 1). In men, the mean BMI, waist circumference, and muscle mass percentage (i.e., ASM/weight) was $24.05 \pm 0.09 \mathrm{~kg} / \mathrm{m}^{2}, 85.1 \pm 0.3 \mathrm{~cm}$, and $31.53 \% \pm 0.13 \%$, respectively. In women, these values were $23.96 \pm 0.1 \mathrm{~kg} / \mathrm{m}^{2}, 80.6 \pm 0.4 \mathrm{~cm}$, and $24.63 \% \pm 0.11 \%$, respectively. The mean sleep duration was $6.9 \pm 0.1$ and $6.7 \pm 0.04 \mathrm{~h} / \mathrm{d}$ in men and women, respectively. In addition, most men (79.2\%) and most women (73.9\%) reported sleeping 6-8 h/d.

The descriptive characteristics of the study population stratified according to sleep duration and sex are shown in Table 2. In men, sleep duration was significantly associated with age, height, body weight, BMI, total body fat percentage, alcohol drinking status, and household income $(\mathrm{P}<0.05$ for each). In women, sleep duration was significantly

Table 1. General characteristics of the study population stratified by sex

\begin{tabular}{lcrr}
\hline \multicolumn{1}{c}{ Characteristic } & Men $(\mathrm{n}=1,542)$ & Women $(\mathrm{n}=1,990)$ & P-value $^{*}$ \\
\hline Age $(\mathrm{y})$ & $53.7 \pm 0.4$ & $55.7 \pm 0.4$ & $<0.001$ \\
Height $(\mathrm{cm})$ & $169.0 \pm 0.2$ & $155.0 \pm 0.2$ & $<0.001$ \\
Body weight $(\mathrm{kg})$ & $68.5 \pm 0.3$ & $57.7 \pm 0.3$ & $<0.001$ \\
Waist circumference $(\mathrm{cm})$ & $85.1 \pm 0.3$ & $80.6 \pm 0.4$ & $<0.001$ \\
Body mass index $\left(\mathrm{kg} / \mathrm{m}^{2}\right)$ & $24.05 \pm 0.09$ & $23.96 \pm 0.1$ & 0.506 \\
Total body fat $(\%)$ & $23.3 \pm 0.3$ & $34.7 \pm 0.2$ & $<0.001$ \\
ASM $(\mathrm{kg})$ & $21.5 \pm 0.1$ & $14.1 \pm 0.1$ & $<0.001$ \\
ASM/body weight $(\%)$ & $31.53 \pm 0.13$ & $24.63 \pm 0.11$ & $<0.001$ \\
Current smoker & $40.7( \pm 1.7)$ & $4.7( \pm 0.7)$ & $<0.001$ \\
Alcohol drinker & $76.4( \pm 1.4)$ & $37.2( \pm 1.4)$ & $<0.001$ \\
Regular exercise & $24.5( \pm 1.5)$ & $21.5( \pm 1.3)$ & 0.074 \\
Lowest household income & $16.2( \pm 1.3)$ & $24( \pm 1.6)$ & $<0.001$ \\
Sleep duration $(\mathrm{h} / \mathrm{d})$ & $6.9 \pm 0.1$ & $6.7 \pm 0.04$ & $<0.001$ \\
Sleep duration $(\mathrm{h} / \mathrm{d})$ & & & $<0.001$ \\
$\leq 5$ & $12.6( \pm 0.9)$ & $19.8( \pm 1.2)$ & \\
$6-8$ & $79.2( \pm 1.2)$ & $73.9( \pm 1.3)$ & \\
$\geq 9$ & $8.3( \pm 1.0)$ & $6.3( \pm 0.9)$ &
\end{tabular}

Values are presented as mean \pm standard error or $\%$ ( \pm standard error). ASM, appendicular skeletal muscle mass.

${ }^{*}$ Calculated using Student t-test or chi-square tests.

Table 2. General characteristics of the study population stratified by sex and self-reported sleep duration

\begin{tabular}{|c|c|c|c|c|c|c|c|c|}
\hline \multirow{2}{*}{ Variable } & \multicolumn{4}{|c|}{ Men } & \multicolumn{4}{|c|}{ Women } \\
\hline & $\leq 5 \mathrm{~h} / \mathrm{d}(\mathrm{n}=216)$ & $6-8 \mathrm{~h} / \mathrm{d}(\mathrm{n}=1,204)$ & $\geq 9 \mathrm{~h} / \mathrm{d}(\mathrm{n}=122)$ & P-value* & $\leq 5 \mathrm{~h} / \mathrm{d}(\mathrm{n}=402)$ & $6-8 \mathrm{~h} / \mathrm{d}(\mathrm{n}=1,461)$ & $\geq 9 \mathrm{~h} / \mathrm{d}(\mathrm{n}=127)$ & P-value* \\
\hline Age (y) & $57.5 \pm 0.8$ & $52.9 \pm 0.4$ & $56.2 \pm 1.1$ & $<0.001$ & $60.8 \pm 0.8$ & $54.1 \pm 0.4$ & $59.5 \pm 1.4$ & $<0.001$ \\
\hline Height (cm) & $168.0 \pm 0.1$ & $169.0 \pm 0.2$ & $167.0 \pm 0.1$ & 0.005 & $154.0 \pm 0.5$ & $156.0 \pm 0.2$ & $154.0 \pm 0.1$ & $<0.001$ \\
\hline Body weight (kg) & $67.8 \pm 0.9$ & $68.9 \pm 0.3$ & $65.2 \pm 1.2$ & 0.007 & $57.1 \pm 0.6$ & $58.0 \pm 0.3$ & $57.1 \pm 0.9$ & 0.257 \\
\hline Waist circumference (cm) & $85.1 \pm 0.8$ & $85.3 \pm 0.3$ & $83.2 \pm 1.0$ & 0.152 & $81.5 \pm 0.5$ & $80.2 \pm 0.4$ & $82.3 \pm 1.5$ & 0.025 \\
\hline Body mass index $\left(\mathrm{kg} / \mathrm{m}^{2}\right)$ & $24.1 \pm 0.28$ & $24.12 \pm 0.09$ & $23.29 \pm 0.31$ & 0.042 & $24.01 \pm 0.19$ & $23.93 \pm 0.11$ & $24.18 \pm 0.4$ & 0.758 \\
\hline Total body fat (\%) & $22.9 \pm 0.5$ & $23.5 \pm 0.3$ & $21.9 \pm 0.7$ & 0.021 & $34.6 \pm 0.4$ & $34.7 \pm 0.2$ & $35.5 \pm 0.6$ & 0.289 \\
\hline ASM $(\mathrm{kg})$ & $21.4 \pm 0.3$ & $21.6 \pm 0.1$ & $20.8 \pm 0.3$ & 0.099 & $14.1 \pm 0.2$ & $14.2 \pm 0.1$ & $13.7 \pm 0.2$ & 0.052 \\
\hline ASM/body weight (\%) & $31.64 \pm 0.25$ & $31.45 \pm 0.13$ & $32.14 \pm 0.36$ & 0.098 & $24.84 \pm 0.18$ & $24.61 \pm 0.11$ & $24.21 \pm 0.26$ & 0.059 \\
\hline Current smoker & $38.2( \pm 3.8)$ & $40.6( \pm 2.0)$ & $46.3( \pm 4.7)$ & 0.404 & $5.8( \pm 1.6)$ & $4.4( \pm 0.8)$ & $4.3( \pm 2.2)$ & 0.695 \\
\hline Alcohol drinker & $67.3( \pm 4.0)$ & $78.5( \pm 1.4)$ & $70.6( \pm 4.8)$ & 0.005 & $30( \pm 2.6)$ & $39.6( \pm 1.6)$ & $31.8( \pm 4.9)$ & 0.005 \\
\hline Regular exercise & $24.8( \pm 3.7)$ & $24.4( \pm 1.7)$ & $25.3( \pm 4.9)$ & 0.979 & $20.3( \pm 2.7)$ & $22.3( \pm 1.6)$ & $16.8( \pm 3.6)$ & 0.448 \\
\hline Lowest household income & $27( \pm 3.6)$ & $14.2( \pm 1.3)$ & $18.5( \pm 4.1)$ & $<0.001$ & $35.4( \pm 3.2)$ & $19.4( \pm 1.5)$ & $41.6( \pm 5.6)$ & $<0.001$ \\
\hline
\end{tabular}

Values are presented as mean \pm standard error or $\%$ ( \pm standard error).

ASM, appendicular skeletal muscle mass.

${ }^{*}$ Calculated using analysis of variance and chi-square tests. 
associated with age, height, waist circumference, alcohol drinking status, and household income $(\mathrm{P}<0.05$ for each).

The results of the logistic regression analyses for the association of sleep duration with obesity and sarcopenia in men are shown Table 3. We found that, in men, sleep duration was not associated with general obesity, central obesity, or sarcopenia before or after adjustment for covariates. Specifically, after adjustment for covariates, the relationship between sleep duration and central obesity was U-shaped, but was not significant (for responders sleeping $\leq 5 \mathrm{~h} / \mathrm{d}$ : OR, 1.08; $95 \% \mathrm{CI}$, $0.72-1.63$; for responders sleeping $\geq 9 \mathrm{~h} / \mathrm{d}$ : OR, 1.30; 95\% CI, 0.78-2.15).

Table 4 presents the results of the logistic regression analyses for the association of sleep duration with obesity and sarcopenia in women. We found that the association between sleep duration and sarcopenia was stronger in women sleeping $\geq 9 \mathrm{~h} / \mathrm{d}$ than in those sleeping $6-8 \mathrm{~h} / \mathrm{d}$ both before (OR, 1.99; 95\% CI, 1.19-3.34) and after (OR, 1.77; 95\% CI, 1.06-2.96) adjustment for covariates. Additionally, we found that, in women, the relationship between sleep duration and general obesity was U-shaped before adjustment for covariates (OR, 1.08; 95\% CI, 0.84-1.40 for women sleeping $\leq 5 \mathrm{~h} / \mathrm{d}$; OR, 1.14; 95\% CI, 0.75-1.74 for women sleeping $\geq 9 \mathrm{~h} / \mathrm{d}$ ), while the relationship between sleep duration and central obesity was U-shaped after adjusting for covariates (OR, 1.11; 95\% CI, 0.85-1.44 for women sleeping $\leq 5 \mathrm{~h} / \mathrm{d}$; OR, 1.02; 95\% CI, 0.61-1.69 for women sleeping $\geq 9 \mathrm{~h} / \mathrm{d}$ ); however, neither relationship was significant.

\section{DISCUSSION}

The purpose of this study was to investigate the association of sleep duration with obesity and sarcopenia in Korean adults $\geq 40$ years of age. In this representative sample of the Korean population, we found that women with longer sleep durations had a higher risk of sarcopenia.

Several studies have investigated the relationships between sleep duration and sarcopenia. One study reported that postmenopausal women with long sleep durations had significantly lower values of skeletal muscle mass, ${ }^{8)}$ while another reported that poor sleep quality was independently associated with physical disability in older adults. ${ }^{18)}$ Moreover, sleep disturbances were associated with poor hand-grip strength, which is a useful indicator of sarcopenia. ${ }^{19)}$ In the present study, we found that longer sleep duration was associated with significantly higher incidence of sarcopenia in Korean women, even after adjusting for potential confounders. However, the exact biological mechanisms underlying the relationship between sleep and sarcopenia remain unclear, and further research is warranted to investigate the related pathophysiologies.

The current body of literature suggests that several mechanisms may contribute to the relationship between sleep and sarcopenia. Previous studies have reported that poor sleep quality and long sleep duration are associated with low physical activity. ${ }^{9,18,20)}$ Indeed, decreased physical activity is considered an important risk factor for sarcopenia. ${ }^{5)}$ Consequently, it is possible that reduced levels of physical activity resulting from long sleep duration can increase the risk for sarcopenia. ${ }^{5,9,20)}$ In addition, some studies have suggested that long sleep duration may result in impaired cognitive function, ${ }^{11)}$ which is reportedly associated with disturbances in physical function, including gait and balance disorders and reduced strength in the lower extremities, thereby increasing the risk for sarcopenia. ${ }^{21)}$ With regard to sleep-related hormonal pathways, poor sleep quality has been associated with decreased secretion of growth hormone, insulin-like growth factor 1 , and testosterone, which results in the deterioration of muscle mass. ${ }^{22,23)}$

Table 3. Association of sleep duration with obesity and sarcopenia in men

\begin{tabular}{|c|c|c|c|c|c|c|}
\hline \multirow{2}{*}{ Variable } & \multicolumn{3}{|c|}{ Unadjusted OR (95\% Cl) } & \multicolumn{3}{|c|}{ Adjusted $^{\star}$ OR $(95 \%$ Cl) } \\
\hline & $\leq 5 \mathrm{~h} / \mathrm{d}$ & $6-8 \mathrm{~h} / \mathrm{d}$ & $\geq 9 \mathrm{~h} / \mathrm{d}$ & $\leq 5 \mathrm{~h} / \mathrm{d}$ & $6-8 \mathrm{~h} / \mathrm{d}$ & $\geq 9 \mathrm{~h} / \mathrm{d}$ \\
\hline General obesity & $0.88(0.60-1.28)$ & 1 & $0.80(0.50-1.26)$ & $0.97(0.65-1.45)$ & 1 & $0.89(0.55-1.42)$ \\
\hline Central obesity & $1.00(0.67-1.49)$ & 1 & $1.25(0.76-2.05)$ & $1.08(0.72-1.63)$ & 1 & $1.30(0.78-2.15)$ \\
\hline Sarcopenia $^{\dagger}$ & $0.82(0.54-1.25)$ & 1 & $0.59(0.32-1.11)$ & $0.78(0.51-1.20)$ & 1 & $0.57(0.31-1.05)$ \\
\hline
\end{tabular}

Associations calculated using multivariate logistic regression analysis.

$\mathrm{OR}$, odds ratio; $\mathrm{Cl}$, confidence interval.

*Adjusted for age, current smoking status, alcohol drinking status, regular exercise status, and household income quartile. 'Sarcopenia was defined as low muscle mass percentage (ratio between appendicular skeletal muscle mass and weight), with values $\leq 29.26 \%$ in men.

Table 4. Association of sleep duration with obesity and sarcopenia in women

\begin{tabular}{|c|c|c|c|c|c|c|}
\hline \multirow{2}{*}{ Variable } & \multicolumn{3}{|c|}{ Unadjusted ORs (95\% Cl) } & \multicolumn{3}{|c|}{ Adjusted ORs (95\% Cl) } \\
\hline & $\leq 5 \mathrm{~h} / \mathrm{d}$ & $6-8 \mathrm{~h} / \mathrm{d}$ & $\geq 9 \mathrm{~h} / \mathrm{d}$ & $\leq 5 \mathrm{~h} / \mathrm{d}$ & $6-8 \mathrm{~h} / \mathrm{d}$ & $\geq 9 \mathrm{~h} / \mathrm{d}$ \\
\hline General obesity & $1.08(0.84-1.40)$ & 1 & $1.14(0.75-1.74)$ & $0.96(0.74-1.23)$ & 1 & $1.01(0.66-1.56)$ \\
\hline Central obesity & $0.79(0.63-1.01)$ & 1 & $0.80(0.47-1.34)$ & $1.11(0.85-1.44)$ & 1 & $1.02(0.61-1.69)$ \\
\hline Sarcopenia ${ }^{\dagger}$ & $0.92(0.67-1.28)$ & 1 & $1.99(1.19-3.34)$ & $0.78(0.56-1.08)$ & 1 & $1.77(1.06-2.96)$ \\
\hline
\end{tabular}

Associations calculated using multivariate logistic regression analysis.

$\mathrm{OR}$, odds ratio; $\mathrm{Cl}$, confidence interval.

*Adjusted for age, current smoking status, alcohol drinking status, regular exercise status, and household income quartile. 'Sarcopenia was defined as low muscle mass percentage (ratio between appendicular skeletal muscle mass and weight), with values $\leq 29.54 \%$ in women. 
Taken together, these findings suggest that general physical illness associated with long sleep duration may increase the risk of sarcopenia.

In the present study, women with longer sleep duration had a higher incidence of sarcopenia, whereas no significant relationship was observed in men. Although the mechanisms underlying this relationship remain unclear, it is possible that there are sex-specific differences in the pathophysiology of health conditions related to sleep duration. One study suggested that sleep duration may be associated with cardiometabolic outcomes, but only in women. ${ }^{24)}$ A recent study conducted in Korea found that long sleep duration was associated with a higher prevalence of chronic kidney disease among Korean women, whereas no significant relationship was noted in men. ${ }^{25)}$ Further detailed studies are needed to examine the exact mechanism of sex-specific disorders related to sleep duration.

To prevent sarcopenia in the general population, it is important to improve sleep quality through adequate physical activity. Physical exercise is the single most important intervention effective in reducing the risk of sarcopenia, and has a positive impact on physical function ${ }^{15}$ and sleep quality. Specifically, regular aerobic or resistance training has been reported to positively influence sleep parameters and sleep disorders, while resistance training has been shown to have acute effects on objective sleep parameters, decreasing the arousal index and possibly leading to higher sleep consolidation. ${ }^{26,27)}$ Therefore, public health interventions are needed in order to develop effective programs for the promotion of regular physical activities and thus improve sleep quality.

The present study had several limitations. First, this was a cross-sectional study; therefore, we were unable to examine the temporal association of sleep duration with obesity and sarcopenia. Therefore, while our findings suggest that reduced levels of physical activity resulting from long sleep duration may lead to sarcopenia, it is also possible that sarcopenia could lead to long sleep duration. Second, when diagnosing sarcopenia, we did not define muscle function in terms of muscle strength or physical performance. Instead, sarcopenia was diagnosed based only on decreased skeletal muscle mass, which may have led to misidentifying participants with or without sarcopenia. Third, the study measured sleep duration based on self-reports, which may not have resulted in accurate data. However, a previous study found that self-reported data on sleep duration were strongly correlated with actigraphy-based measurements. ${ }^{28)}$

To the best of our knowledge, this study is the first to report an association between sleep duration and sarcopenia in a Korean population. Moreover, our findings were based on a large sample that was highly representative of the general population of Korea, and all analyses used sample weights and adjusted for the complex sample design of the survey.

Therefore, despite the limitations, our study provides evidence that longer sleep duration is associated with a significantly higher incidence of sarcopenia in Korean women over 40 years of age. In the future, large-scale cohort studies should focus on determining causality in the association of sleep duration with obesity and sarcopenia, and on exploring the possible mechanisms of these relationships. As sleep duration is a potentially modifiable risk factor, our results may help health advisors develop strategies to prevent or delay the progression of sarcopenia.

\section{CONFLICT OF INTEREST}

No potential conflict of interest relevant to this article was reported.

\section{REFERENCES}

1. Grimby G, Saltin B. The ageing muscle. Clin Physiol 1983;3:209-18.

2. Jang HC. Recent progression in sarcopenia and sarcopenic obesity. J Korean Geriatr Soc 2011;15:1-7.

3. Houston DK, Nicklas BJ, Zizza CA. Weighty concerns: the growing prevalence of obesity among older adults. J Am Diet Assoc 2009;109:1886-95.

4. Janssen I, Heymsfield SB, Ross R. Low relative skeletal muscle mass (sarcopenia) in older persons is associated with functional impairment and physical disability. J Am Geriatr Soc 2002;50:889-96.

5. Fielding RA, Vellas B, Evans WJ, Bhasin S, Morley JE, Newman AB, et al. Sarcopenia: an undiagnosed condition in older adults: current consensus definition: prevalence, etiology, and consequences. International working group on sarcopenia. J Am Med Dir Assoc 2011;12:24956.

6. Vitiello MV. Sleep disorders and aging: understanding the causes. J Gerontol A Biol Sci Med Sci 1997;52:M189-91.

7. Bonnet MH, Arand DL. We are chronically sleep deprived. Sleep 1995;18:908-11.

8. Fex A, Barbat-Artigas S, Dupontgand S, Filion ME, Karelis AD, Aubertin-Leheudre M. Relationship between long sleep duration and functional capacities in postmenopausal women. J Clin Sleep Med 2012;8:309-13.

9. Bellavia A, Akerstedt T, Bottai M, Wolk A, Orsini N. Sleep duration and survival percentiles across categories of physical activity. Am J Epidemiol 2014;179:484-91.

10. Bae D, Wickrama KA, O'Neal CW. Social consequences of early socioeconomic adversity and youth BMI trajectories: gender and race/ethnicity differences. J Adolesc 2014;37:883-92.

11. Gildner TE, Liebert MA, Kowal P, Chatterji S, Snodgrass JJ. Associations between sleep duration, sleep quality, and cognitive test performance among older adults from six middle income countries: results from the Study on Global Ageing and Adult Health (SAGE). J Clin Sleep Med 2014;10:613-21.

12. Park SE, Kim HM, Kim DH, Kim J, Cha BS, Kim DJ. The association between sleep duration and general and abdominal obesity in Koreans: data from the Korean National Health and Nutrition Examination Survey, 2001 and 2005. Obesity (Silver Spring) 2009;17:767-71.

13. Ministry of Health and Welfare, Korea Centers for Disease Control and Prevention. 2010 Korea health statistics: Korean National Health and Nutrition Examination Survey (KNHANES V-1). Cheongju: Korea Centers for Disease Control and Prevention; 2011 [cited 2017 Feb 20]. Available from: http://knhanes.cdc.go.kr/knhanes.

14. Lee S, Park HS, Kim SM, Kwon HS, Kim DY, Kim DJ, et al. Cut-off points 
of waist circumference for defining abdominal obesity in the Korean population. Korean J Obes 2006;15:1-9.

15. Chen LK, Liu LK, Woo J, Assantachai P, Auyeung TW, Bahyah KS, et al. Sarcopenia in Asia: consensus report of the Asian Working Group for Sarcopenia. J Am Med Dir Assoc 2014;15:95-101.

16. Cappuccio FP, Taggart FM, Kandala NB, Currie A, Peile E, Stranges S, et al. Meta-analysis of short sleep duration and obesity in children and adults. Sleep 2008;31:619-26.

17. Ramos AR, Wallace DM, Pandi-Perumal SR, Williams NJ, Castor C, Sevick MA, et al. Associations between sleep disturbances and diabetes mellitus among blacks with metabolic syndrome: results from the Metabolic Syndrome Outcome Study (MetSO). Ann Med 2015;47:2337.

18. Chien MY, Chen HC. Poor sleep quality is independently associated with physical disability in older adults. J Clin Sleep Med 2015;11:22532.

19. Vasconcelos KS, Dias JM, Bastone Ade C, Vieira RA, Andrade AC, Perracini MR, et al. Handgrip strength cutoff points to identify mobility limitation in community-dwelling older people and associated factors. J Nutr Health Aging 2016;20:306-15.

20. Patel SR, Malhotra A, Gottlieb DJ, White DP, Hu FB. Correlates of long sleep duration. Sleep 2006;29:881-9.

21. Narici MV, Maffulli N. Sarcopenia: characteristics, mechanisms and functional significance. Br Med Bull 2010;95:139-59.

22. Bhasin S, Storer TW, Berman N, Callegari C, Clevenger B, Phillips J, et al. The effects of supraphysiologic doses of testosterone on muscle size and strength in normal men. N Engl J Med 1996;335:1-7.

23. Balbo M, Leproult R, Van Cauter E. Impact of sleep and its disturbances on hypothalamo-pituitary-adrenal axis activity. Int J Endocrinol 2010;2010:759234.

24. Miller MA, Kandala NB, Kivimaki M, Kumari M, Brunner EJ, Lowe GD, et al. Gender differences in the cross-sectional relationships between sleep duration and markers of inflammation: Whitehall II study. Sleep 2009;32:857-64.

25. Choi H, Kim HC, Lee JY, Lee JM, Choi DP, Suh I. Sleep duration and chronic kidney disease: the Korean Genome and Epidemiology Study (KoGES)-Kangwha study. Korean J Intern Med 2017;32:323-34.

26. Yang PY, Ho KH, Chen HC, Chien MY. Exercise training improves sleep quality in middle-aged and older adults with sleep problems: a systematic review. J Physiother 2012;58:157-63.

27. Viana VA, Esteves AM, Boscolo RA, Grassmann V, Santana MG, Tufik S, et al. The effects of a session of resistance training on sleep patterns in the elderly. Eur J Appl Physiol 2012;112:2403-8.

28. Lockley SW, Skene DJ, Arendt J. Comparison between subjective and actigraphic measurement of sleep and sleep rhythms. J Sleep Res 1999;8:175-83. 